\title{
Folate supplementation increases genomic DNA methylation in the liver of elder rats
}

\author{
Sang-Woon Choi ${ }^{1}$, Simonetta Friso ${ }^{2}$, Mary K. Keyes ${ }^{1}$ and Joel B. Mason ${ }^{1,3}$ \\ ${ }^{1}$ Vitamins and Carcinogenesis Laboratory, Jean Mayer US Department of Agriculture Human Nutrition Research Center on Aging at Tufts \\ University, Washington Street, Boston, MA 02111, USA \\ ${ }^{2}$ Department of Clinical and Experimental Medicine, Policlinico G. B. Rossi, University of Verona, 37134 Verona, Italy \\ ${ }^{3}$ Divisions of Clinical Nutrition and Gastroenterology, Tufts University School of Medicine, Boston, MA 02111, USA
}

(Received 12 April 2004 - Revised 24 August 2004 - Accepted 26 August 2004)

\begin{abstract}
The availability of folate is implicated as a determinant of DNA methylation, a functionally important feature of DNA. Nevertheless, when this phenomenon has been examined in the rodent model, the effect has not always been observed. Several reasons have been postulated for the inconsistency between studies: the rodent is less dependent on folate as a methyl source than man; juvenile animals, which most studies use, are more resistant to folate depletion than old animals; methods to measure genomic DNA methylation might not be sensitive enough to detect differences. We therefore examined the relationship between folate and genomic DNA methylation in an elder rat model with a newly developed method that can measure genomic DNA methylation sensitively and precisely. Thirty-nine 1-year-old rats were divided into three groups and fed a diet containing $0,4.5$ or $18 \mu \mathrm{mol}$ folate/kg (folate-deplete, -replete and supplemented groups, respectively). Rats were killed at 8 and 20 weeks. At both time points, mean liver folate concentrations increased incrementally between the folate-deplete, -replete and -supplemented rats $(P$ for trend $<0.001)$ and by 20 weeks hepatic DNA methylation also increased incrementally between the folate-deplete, -replete and -supplemented rats $(P$ for trend $=0.025)$. At both time points folate-supplemented rats had significantly increased levels of DNA methylation compared with folate-deplete rats $(P<0.05)$. There was a strong correlation between hepatic folate concentration and genomic DNA methylation in the liver $(r 0.48, P=0.004)$. In the liver of this animal model, dietary folate over a wide range of intakes modulates genomic DNA methylation.
\end{abstract}

Folate: DNA methylation: Ageing: Liver: Rat

In vertebrate genomes, approximately $4 \%$ of cytosine residues are modified post-synthetically to 5-methylcytosine. Most of these 5-methylcytosine residues are found in the $\mathrm{CpG}$ dinucleotide sequence within promoter regions. Maintenance of normal patterns of DNA methylation is important for cellular homeostasis since DNA methylation is thought to play a critical role in both the regulation of gene expression and the maintenance of gene integrity (Antequera et al. 1989; Razin \& Cedar, 1991).

In some of its coenzymatic forms folate is a biological methyl donor, and thus it appears to be a determinant of genomic DNA methylation under certain circumstances. In human studies conducted in metabolic units, folate depletion has consistently been observed to produce genomic DNA hypomethylation in peripheral blood lymphocytes. Jacob et al. (1998) reported that marginally folate-deplete volunteers housed in a metabolic unit had diminished genomic DNA methylation. Subsequent repletion of folate status reversed the effect. Rampersaud et al. (2000) reported a similar effect in their metabolic unit study where a less restrictive diet $(120 \mu \mathrm{g}$ folate/d) was used.

In 1993 Balaghi et al. demonstrated similar effects in laboratory rodents; hepatic DNA from folate-deficient rats became genomically hypomethylated compared with DNA from control rats. However, when this phenomenon was re-examined in rat liver (Kim et al. 1995) or in other tissues such as the colon (Sohn et al. 2003), the effect was not always reproduced. Although the different degrees of folate depletion achieved in these studies have sometimes been cited to explain these discrepancies, other factors may be involved as well.

Ageing has also been observed to be associated with DNA hypomethylation. Studies in both man and laboratory rodents have repeatedly observed incremental decreases in genomic DNA methylation with ageing, and such effects have been observed in a wide variety of tissues (Mays-Hoopes et al. 1986; Wilson et al. 1987; Drinkwater et al. 1989). Even in cell culture, repeated passages (to the extent that it is a model of ageing) are associated with decreases in DNA methylation (Wilson \& Jones, 1983; Holliday, 1986).

We therefore investigated the effect of folate status on genomic DNA methylation in a well-established rat model of chronic moderate folate depletion (Varela-Moreiras \& Selhub, 1992; Kim et al. 1996) in elder rats. This model of chronic moderate deficiency is more clinically relevant than the short-term, severe deficiency described in previous studies (Choi et al. 1998). It is relevant to note that this long-term, moderate deficiency has not been found 
in prior studies of juvenile adult rodents to be a sufficiently perturbing force to induce a significant degree of hypomethylation in hepatic DNA (Kim et al. 1995, 1996).

\section{Experimental methods}

\section{Animals}

The present study was approved by the Institutional Animal Care and Use Committee of the Jean Mayer US Department of Agriculture Human Nutrition Research Center on Aging at Tufts University. Middle-aged (12 months old, $n$ 39) male Sprague-Dawley rats (Zivic-Miller, Zelienople, PA, USA) were randomized to receive one of three diets identical with regard to amino acids but with different folate levels: (i) $0 \mu \mathrm{mol}$ folate $/ \mathrm{kg}$ (folate-deplete); (ii) $4.5 \mu \mathrm{mol} / \mathrm{kg}$ (basal requirement of folate); (iii) $18 \mu \mathrm{mol} / \mathrm{kg}$ (folate-supplemented state) (Dyets, Bethlehem, PA, USA). These amino acid defined diets constitute a standard means of predictably inducing deplete, replete and supplemented levels of folate status in rodents (Walzem \& Clifford, 1988; Bills et al. 1991). By intent, a moderate, and not a severe, degree of folate depletion is produced in the deplete groups owing to the fact that no sulphonamide is added to their diets.

Rats were housed individually in wire-bottomed stainless steel cages to minimize coprophagy. Body weights were recorded biweekly. Water was supplied ad libitum. The amount of diet supplied to each diet group was matched to the mean daily food consumption of the group with the least food consumption. At 8 weeks after initiation of the diets, five rats from each group were killed. At 20 weeks, at which time the animals were 17 months of age, eight rats from each group were killed. The time points of 8 and 20 weeks were chosen because we wished to examine the effects of chronic depletion and because our prior studies had demonstrated that a mild and moderate degree of depletion occurs at these two time points, respectively.

Rats were anaesthetized in a $\mathrm{CO}_{2}$ breathing chamber and the abdomen was then opened. The liver was harvested and the animal then killed by cross-cutting the aorta. Aliquots of liver tissue were flash frozen in liquid $\mathrm{N}$ and subsequently stored at $-70^{\circ} \mathrm{C}$.

\section{Measurement of folate concentration and S-adenosylhomocysteine}

Hepatic folate concentrations were determined by a conventional microbiological microtitre plate assay using Lactobacillus casei after treatment with chicken pancreas conjugase (Tamura, 1990). Hepatic $S$-adenosylhomocysteine (SAdoHcy) concentrations were measured by HPLC with UV detection using the method described Fell et al. (1985).DNA methylation

Liquid chromatography-electrospray ionization MS (Hewlett Packard/Bruker, Billerica, MA, USA) was used to analyse genomic DNA methylation. A detailed method has been previously described and validated by us (Friso et al. 2002a,b).

Briefly, DNA was extracted by a conventional technique using a lysis buffer containing proteinase $\mathrm{K}$ followed by extraction with phenol, chloroform and isoamyl alcohol. Then $1 \mu \mathrm{g}$ DNA was hydrolysed by sequential digestion with three enzymes (Crain, 1990): nuclease P1 (Roche Molecular Biochemicals, Mannheim, Germany); venom phosphodiesterase I (Sigma, St. Louis, MO, USA); alkaline phosphatase (Sigma). The hydrolysed DNA sol- ution was delivered directly onto the analytical column (Supelco, Bellefonte, PA, USA) in isocratic mode. This allowed the separation of the four DNA bases as well as the identification of 5-methylcytosine. Electrospray ionization MS was performed in positive ion mode. Identification of cytosine and 5-methylcytosine was obtained by mass spectrometric analysis of chromatographic peaks. The isotopomers $\left[{ }^{15} \mathrm{~N}_{3}\right] 2^{\prime}$-deoxycytidine and methyl-D3, ring-6-D1 5-methyl-2'-deoxycytidine (Cambridge Isotope Laboratories, Cambridge, MA, USA) were used as internal standards. DNA methylation status was defined as the percentage of 5-methylcytosine of the overall amount of cytosine and 5-methylcytosine.

\section{Statistical analysis}

Statistical significance for differences in quantitative variables was analysed by ANOVA followed by the post hoc Tukey test. Logarithmic transformation was performed on all skewed variables to normalize their distributions and statistical comparison was based on logarithmic-transformed data. $P$ for trend was analysed by regression analysis. For genomic DNA methylation, the difference was examined by a two-tailed Student's $t$ test. The level of significance was set at $P<0.05$ for all analyses. Statistical analysis was performed using Systat 10 for Windows software (SPSS, Chicago, IL, USA).

\section{Results}

We reported some of the data on body weight in a previous paper that focused on the effects of age on colonic folate metabolism (Choi et al. 2003). Rats fed folate-deplete diets experienced a small, but significant, mean weight loss compared with folatereplete and -supplemented rats, starting at 10 weeks (data not shown). All rats survived the 20-week experiment except two elderly rats from the folate-deplete group. They died at 8 and 19 weeks.

Table 1 shows that mean liver folate concentrations increased in an incremental fashion between the folate-deplete, -replete and -supplemented rats $(P$ for trend $<0 \cdot 001)$. This confirms the ability of these diets to produce predictable levels of tissue folate. Furthermore, at both time points the mean liver folate concentrations of the folate-deplete group were significantly lower than the corresponding values of the folate-replete and -supplemented groups $(P<0.05$, Table 1$)$, demonstrating a significant degree of hepatic depletion of folate. In a similar but reciprocal fashion, hepatic SAdoHcy increased incrementally within each group as revealed by the comparison of folate-supplemented, -replete and -deplete rats $(P$ for trend $<0 \cdot 001$, see Table 1). Both hepatic folate and SAdoHcy concentrations of the elder rats were within the same range of values observed in corresponding diet groups of younger adult rats reported in our previous studies (Kim et al. 1995, 1997).

By 20 weeks, the incremental increase in hepatic DNA methylation between the folate-deplete, -replete and -supplemented groups (Fig. 1) was sufficiently prominent to be a significant trend across the entire range of folate status $(P=0.025)$. At both 8 and 20 weeks, folate-supplemented rats had significantly increased hepatic DNA methylation compared with the folate-deplete group $(P<0 \cdot 05)$. A moderately strong, and highly significant, correlation between hepatic folate concentration and genomic DNA methylation $(r 0.48, P=0.004)$ was observed (Fig. 2). 
Table 1. Liver folate and S-adenosylhomocysteine (S-AdoHcy)

(Values are means and standard deviations)

\begin{tabular}{|c|c|c|c|c|c|}
\hline \multirow[b]{2}{*}{ Group } & \multirow[b]{2}{*}{$n$} & \multicolumn{2}{|c|}{ Folate $(\mathrm{nmol} / \mathrm{g})$} & \multicolumn{2}{|c|}{ S-AdoHcy $(\mathrm{nmol} / \mathrm{g})$} \\
\hline & & Mean & SD & Mean & SD \\
\hline 8-week folate-deplete & 5 & $3 \cdot 0^{\mathrm{a}}$ & 0.9 & $14 \cdot 0^{\mathrm{a}}$ & 3.4 \\
\hline 8-week folate-replete & 5 & $15 \cdot 0^{\mathrm{b}}$ & $2 \cdot 0$ & $6 \cdot 9^{b}$ & 1.9 \\
\hline 8-week folate-supplemented & 5 & $20 \cdot 2^{c}$ & $3 \cdot 2$ & $5 \cdot 4^{\mathrm{b}}$ & 1.5 \\
\hline$P$ for trend & & \multicolumn{2}{|c|}{$<0.001$} & \multicolumn{2}{|c|}{$<0.001$} \\
\hline 20-week folate-deplete & 6 & $1 \cdot 8^{\mathrm{a}}$ & 0.7 & $15 \cdot 0^{\mathrm{a}}$ & $7 \cdot 1$ \\
\hline 20-week folate-replete & 8 & $15 \cdot 9^{b}$ & $5 \cdot 2$ & $6 \cdot 2^{b}$ & $2 \cdot 0$ \\
\hline 20-week folate-supplemented & 8 & $22 \cdot 0^{\mathrm{b}}$ & 8.4 & $4 \cdot 4^{\mathrm{b}}$ & $2 \cdot 4$ \\
\hline$P$ for trend & & \multicolumn{2}{|c|}{$<0.001$} & \multicolumn{2}{|c|}{$<0.001$} \\
\hline
\end{tabular}

${ }^{a, b, c}$ Mean values within a column with unlike superscript letters were significantly different $(P<0.05$ by ANOVA followed by the post hoc Tukey multiple comparison test).

\section{Discussion}

Compared with human studies (Jacob et al. 1998; Rampersaud et al. 2000), prior rodent studies of isolated dietary folate deficiency have generally failed to induce genomic DNA hypomethylation (Kim et al. 1995, 1997) with the exception of one study (Balaghi et al. 1993), even though folate depletion has been shown consistently to induce a marked increase in hepatic SAdoHcy, a compound well known to inhibit methylation reactions (De Cabo et al. 1995). It is worth noting that the rodent liver possesses a particularly active alternative pathway by which homocysteine can be remethylated (via the conversion of betaine to dimethylglycine), which may compensate for diminished folate availability and therefore explain, in part, the resistance of the rat liver to develop genomic hypomethylation. Furthermore, discrepancies between prior rodent studies might be ascribable to the different levels of severity or duration of the folate-deficient state that have been studied. There has also been speculation that previous methods to measure genomic DNA methylation might not be sensitive enough to detect the alteration induced by folate depletion due to the imprecision of measurement (Friso et al. 2002b).

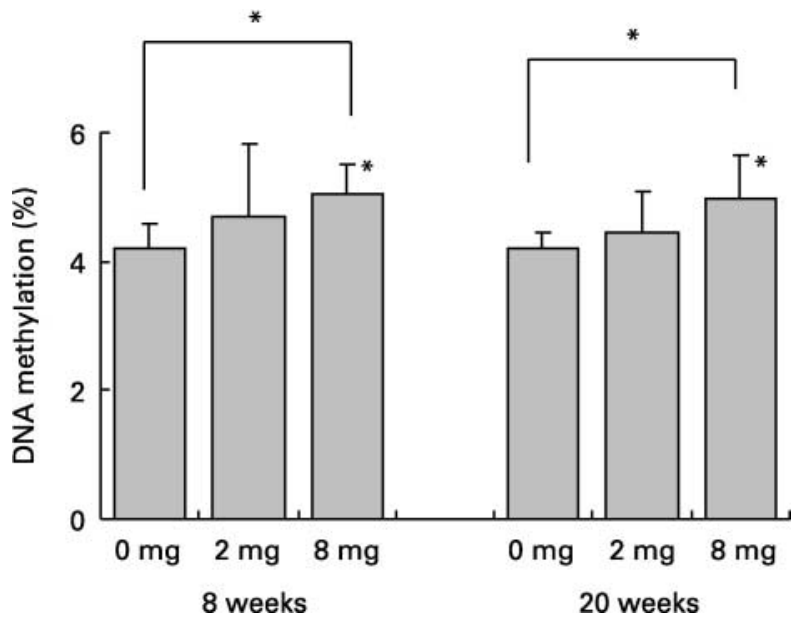

Fig. 1. Hepatic DNA methylation at different levels of dietary folate. By 8 and 20 weeks, hepatic DNA methylation showed a stepwise increase from the folate-deplete group to the folate-replete group to the folate-supplemented group ( $P$ for trend $=0.08$ and 0.025 , respectively). Mean values of DNA methylation (folate-supplemented rats $v$. folate-deplete rats) were significantly different at both 8 weeks (five animals per group) and 20 weeks (eight animals per group): ${ }^{*} P<0.05$.
In the present study, using an elder rat model of chronic folate deficiency and a new, highly precise liquid chromatography/MS method, folate supplementation at four times the basal requirement of the rat (Reeves et al. 1993) significantly increased genomic DNA methylation compared with folate-deplete status in the liver. It is worth emphasizing that folate-deplete rats did not develop significantly lower levels of genomic DNA methylation compared with folate-replete rats, underscoring the importance of examining this phenomenon over a wide range of folate intakes. DNA methylation in the liver of the elder rats also displayed dependence on dietary folate in a significant manner as early as 8 weeks on the diet, an effect that was even more evident at 20 weeks.

One-carbon metabolism is a network of interrelated biochemical reactions in which a one-carbon unit from a donor compound is transferred to tetrahydrofolate for subsequent transfer to other compounds. Folate coenzymes in mammalian tissues thereby act as acceptors or donors of one-carbon units in a variety of reactions involved in amino acid and nucleotide metabolism (Mackenzie, 1984). Within the scope of this function is the synthesis of $S$-adenosylmethionine, a universal methyl donor for several biological methylation reactions. Methionine, the precursor of $S$-adenosylmethionine, is regenerated from homocysteine by methionine synthase in a reaction wherein 5-methyltetrahydrofolate serves

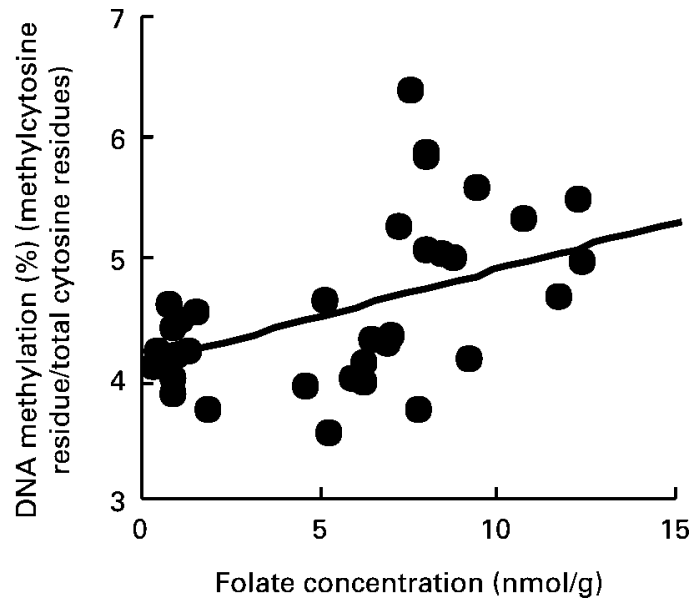

Fig. 2. Correlation between hepatic folate concentration and genomic DNA methylation in the rat liver at 8 and 20 weeks $(n 3)$. A significant correlation was observed ( $r 0.48, P=0.004)$. 
both as a cofactor and a substrate. Reduced availability of 5-methyltetrahydrofolate, the main circulating form of folate, decreases the biosynthesis of $S$-adenosylmethionine, limiting the availability of methyl groups for methylation reactions, and increases the cellular concentration of SAdoHcy, which is a direct inhibitor of methylation reactions. Studies to date suggest that it is the rise in SAdoHcy rather than the decease in $S$-adenosylmethionine that is the more critical determinant of DNA methylation (Yi et al. 2000).

Recent observations in a cystathionine- $\beta$-synthase knockout mouse model (Caudill et al. 2001; Choumenkovitch et al. 2002) as well as in a human study (Yi et al. 2000) have indicated that an elevation in SAdoHcy is sufficient to produce DNA hypomethylation, although the effect is tissue-specific. In the present study, elevations in hepatic SAdoHcy concentration accompanied folate depletion and, as would be expected, SAdoHcy decreased stepwise across the dietary folate levels from depletion to supplementation. This stepwise decrease in SAdoHcy was accompanied by a stepwise increase in genomic DNA methylation status. Therefore, the decrement in hepatic SAdoHcy produced by folate supplementation in this study was probably sufficient to reduce the inhibitory effect of SAdoHcy on genomic DNA methylation.

The identification of $\mathrm{CpG}$ methylation as a mechanism of epigenetic inheritance has led to the speculation that it might be involved in the ageing process (Holliday, 1987), because genomic DNA hypomethylation has also been observed in elder tissues in vivo (Mays-Hoopes et al. 1986; Wilson et al. 1987; Drinkwater et al. 1989). Since some studies indicate that hypomethylation is a consistent feature of proliferating cells (Hoal-van Helden \& van Helden, 1989; Goodman \& Counts, 1993) and increased proliferation is a characteristic of some elder tissues (Ronucci et al. 1988), this provides a possible explanation for this phenomenon. Whether folate depletion might exacerbate this age-related loss of methyl group is unclear, but this possibility is an attractive explanation of how diminished folate status and age might act synergistically to enhance the risk of carcinogenesis.

It is therefore not surprising that folate supplementation increased genomic DNA methylation in the elder rat liver, because folate has been shown in both animal and human studies to have a substantial impact on DNA methylation (Friso \& Choi, 2002) and ageing enhances the susceptibility of folate depletion (Choi et al. 2003). We speculate that folate supplementation diminishes the tendency towards DNA hypomethylation that is produced by the synergy of ageing and lesser amounts of folate in the diet, both of which are important risk factors for carcinogenesis.

In conclusion, dietary folate over a wide range of intakes modulates genomic DNA methylation in the elder rat liver. In particular, folate supplementation at four times the basal requirement significantly increases genomic DNA methylation compared with folate-deplete status. Our ability to detect such an effect was probably related to the age of the animals used and/or the heightened accuracy of the method utilized to measure methylation.

\section{Acknowledgements}

This material is based upon work supported by the US Department of Agriculture, under agreement no. 581950-9-001. Any opinions, findings, conclusion or recommendations expressed in this publication are those of the author(s) and do not necessarily reflect the view of the US Department of Agriculture.

This project was supported in part by an NCI grant (RO3 CA96004-02, S.W.C), a Jean Mayer US Department of Agriculture Human Nutrition Research Center grant (S. W. C.) and NIH grants (K05 CA100048 and U54 CA100971, J. B. M.).

\section{References}

Antequera F, Macleod D \& Bird AP (1989) Specific protection of methylated CpGs in mammalian nuclei. Cell 58, 509-517.

Balaghi M, Horne DW \& Wagner C (1993) Hepatic one-carbon metabolism in early folate deficiency in rats. Biochem $J$ 291, 145-149.

Bills ND, Jones AD \& Clifford AJ (1991) Biological activity of racemic folate mixtures fed to folate-depleted rats. J Nutr 121, 1643-1648.

Caudill MA, Wang JC, Melnyk S, Pogribny IP, Jernigan S, Collins MD, Santos-Guzman J, Swendseid ME, Cogger EA \& James SJ (2001) Intracellular $S$-adenosylhomocysteine concentrations predict global DNA hypomethylation in tissues of methyl-deficient cystathionine beta-synthase heterozygous mice. J Nutr 131, 2811-2818.

Choi SW, Kim YI, Weitzel JN \& Mason JB (1998) Folate depletion impairs DNA excision repair in the colon of the rat. Gut 43, 93-99.

Choi SW, Friso S, Dolnikowski GG, Bagley PJ, Edmondson AN, Smith DE \& Mason JB (2003) Biochemical and molecular aberrations in the rat colon due to folate depletion are age-specific. J Nutr 133, 1206-1212.

Choumenkovitch SF, Selhub J, Bagley PJ, Maeda N, Nadeau MR, Smith DE \& Choi SW (2002) In the cystathionine beta-synthase knockout mouse, elevations in total plasma homocysteine increase tissue $S$-adenosylhomocysteine, but responses of $S$-adenosylmethionine and DNA methylation are tissue specific. J Nutr 132, 2157-2160.

Crain PF (1990) Preparation and enzymatic hydrolysis of DNA and RNA for mass spectrometry. Methods Enzymol 193, 782-790.

De Cabo SF, Santos J \& Fernandez-Piqueras J (1995) Molecular and cytological evidence of $S$-adenosyl-L-homocysteine as an innocuous undermethylating agent in vivo. Cytogenet Cell Genet 71, 187-192.

Drinkwater RD, Blake TJ, Morley AA \& Turner DR (1989) Human lymphocytes aged in vivo have reduced levels of methylation in transcriptionally active and inactive DNA. Mutat Res 219, 29-37.

Fell D, Benjamin LE \& Steele RD (1985) Determination of adenosine and $S$-adenosyl derivatives of sulfur amino acids in rat liver by high performance liquid chromatography. J Chromatogr 345, 150-156.

Friso S \& Choi SW (2002) Gene-nutrient interactions and DNA methylation. J Nutr 132, Suppl. 8, 2382S-2387S.

Friso S, Choi SW, Girelli D, et al. (2002a) A common mutation in the 5,10-methylenetetrahydrofolate reductase gene affects genomic DNA methylation through an interaction with folate status. Proc Natl Acad Sci USA 99, 5606-5611.

Friso S, Choi SW, Dolnikowski GG \& Selhub J (2002b) A new method to assess genomic DNA methylation using high performance liquid chromatography-electrospray ionization mass spectrometry. Anal Chem 74, 4526-4531.

Goodman JI \& Counts JL (1993) Hypomethylation of DNA: a possible nongenotoxic mechanism underlying the role of cell proliferation in carcinogenesis. Environ Health Perspect 101, Suppl. 5, 169-172.

Hoal-van Helden EG \& van Helden PD (1989) Age-related methylation changes in DNA may reflect the proliferative potential of organs. Mutat Res 219, 263-266.

Holliday R (1986) Strong effects of 5-azacytidine on the in vitro lifespan of human diploid fibroblasts. Exp Cell Res 166, 543-552.

Holliday R (1987) The inheritance of epigenetic defects. Science 238, $163-170$.

Jacob RA, Gretz DM, Taylor PC, James SJ, Pogribny IP, Miller BJ, Henning SM \& Swendseid ME (1998) Moderate folate depletion increases plasma homocysteine and decreases lymphocyte DNA methylation in postmenopausal women. J Nutr 128, 1204-1212. 
Kim YI, Christmas JK, Fleet JC, Cravo ML, Salomon RN, Smith D, Ordovas J, Selhub J \& Mason JB (1995) Moderate folate deficiency does not cause global hypomethylation of hepatic and colonic DNA or c-myc-specific hypomethylation of colonic DNA in rats. Am J Clin Nutr 61, 1083-1090.

Kim YI, Salomon RN, Graeme-Cook F, Choi SW, Smith DE, Dallal GE \& Mason JB (1996) Dietary folate protects against the development of macroscopic colonic neoplasms in a dose-responsive manner in rats. Gut 39, 732-740.

Kim YI, Pogribny IP, Basnakian AG, Miller JW, Selhub J, James SJ \& Mason JB (1997) Folate deficiency in rats induces DNA strand breaks and hypomethylation within the p53 tumor suppressor gene. Am J Clin Nutr 65, 46-52.

MacKenzie RE (1984) Biogenesis and interconversion of substituted tetrahydrofolates. In Folate and Pterins, vol. 1, pp. 255-306 [RL Blakley and SJ Benkovic, editors]. New York: Wiley.

Mays-Hoopes L, Chao W, Butcher HC \& Huang RC (1986) Decreased methylation of the major mouse long interspersed repeated DNA during aging and in myeloma cells. Dev Genet 7, 65-73.

Rampersaud GC, Kauwell GP, Hutson AD, Cerda JJ \& Bailey LB (2000) Genomic DNA methylation decreases in response to moderate folate depletion in elderly women. Am J Clin Nutr 72, 998-1003.

Razin A \& Cedar H (1991) DNA methylation and gene expression. Microbiol Rev 55, 451-458.

Reeves PG, Nielsen FH \& Fahey GC Jr (1993) AIN-93 purified diets for laboratory rodents: final report of the American Institute of Nutrition ad hoc writing committee on the reformulation of the AIN-76A rodent diet. J Nutr 123, 1939-1951.

Ronucci L, Ponz de Leon M, Scalmati A, Malagoli G, Pratissoli S, Perini M \& Chahin NJ (1988) The influence of age on colonic epithelial cell proliferation. Cancer 62, 2373-2377.

Sohn KJ, Stempak JM, Reid S, Shirwadkar S, Mason JB \& Kim YI (2003) The effect of dietary folate on genomic and p53-specific DNA methylation in rat colon. Carcinogenesis 24, 81-90.

Tamura T (1990) Microbiological assay of folate. In Folic Acid Metabolism in Health and Disease, 1st ed. vol. 13, pp. 121-137 [MF Picciano, ELR Stokstad and JF GregoryIII, editors]. New York: Wiley-Liss.

Varela-Moreiras G \& Selhub J (1992) Long-term folate deficiency alters folate content and distribution differentially in rat tissues. $J$ Nutr 122, 986-991.

Walzem RL \& Clifford AJ (1988) Folate deficiency in rats fed diets containing free amino acids or intact proteins. J Nutr 118, 1089-1096.

Wilson VL \& Jones PA (1983) DNA methylation decreases in aging but not in immortal cells. Science 20, 1055-1057.

Wilson VL, Smith RA, Ma S \& Cutler RG (1987) Genomic 5-methyldeoxycytidine decreases with age. J Biol Chem 262, 9948-9951.

Yi P, Melnyk S, Pogribna M, Pogribny IP, Hine RJ \& James SJ (2000) Increase in plasma homocysteine associated with parallel increases in plasma $S$-adenosylhomocysteine and lymphocyte DNA hypomethylation. J Biol Chem 275, 29318-29323. 